\title{
Loop effect in the coherent neutrino-nucleus scattering
}

\author{
Tong $\mathbf{L i}^{a}$ and Jiajun $\mathbf{L i a o}^{b}$ \\ ${ }^{a}$ School of Physics, Nankai University, \\ Tianjin 300071, China \\ ${ }^{b}$ School of Physics, Sun Yat-Sen University, \\ Guangzhou 510275, China \\ E-mail: litong@nankai.edu.cn, liaojiajun@mail.sysu.edu.cn
}

ABSTRaCT: A connection between the neutrino and an exotic fermion is described in the general neutrino model. In this model the neutrinos can convert into the new fermion and thus the interaction leads to novel recoil spectrum in the neutrino scattering experiments. We study the general neutrino interaction by evaluating both the tree-level and looplevel contributions to the coherent elastic neutrino-nucleus scattering. We illustrate the scattering by taking the framework of a simplified neutrino model with a Dirac fermion $\chi$ and a spin- 0 mediator. For the CP phase in the quark sector being 0 and $\pi / 2$, the detection processes are dominated by the tree-level and loop-level contribution, respectively. We investigate the constraints on the couplings between the mediator and the new particle $\chi$ or the quarks by fitting to the COHERENT data. The parameter space with $m_{\chi}$ larger than the maximal energy of incoming neutrinos can be also constrained by including the loop-level contribution.

Keywords: Beyond Standard Model, Neutrino Physics

ArXiv EPrint: 2008.00743 


\section{Contents}

1 Introduction 1

2 Simplified neutrino model with an exotic fermion 2

3 Coherent elastic neutrino-nucleus scattering 3

4 Other constraints $\quad 9$

4.1 Flavor constraint 9

$\begin{array}{lll}4.2 & \text { LHC constraint } & 10\end{array}$

$\begin{array}{lll}4.3 & \text { Long-lived hypothesis } & 11\end{array}$

5 Conclusions 11

A Loop diagram calculation in neutrino-nucleus scattering 12

\section{Introduction}

The COHERENT experiment has observed the coherent elastic neutrino-nucleus scattering (CE $\nu \mathrm{NS}$ ) process at the $6.7 \sigma$ level [1]. The neutrinos measured at COHERENT are produced at the Spallation Neutron Source (SNS) by stopped pion and muon decays with energies $E \lesssim 53 \mathrm{MeV}$. The $\mathrm{CE} \nu \mathrm{NS}$ process occurs when the moment transfer in the neutrinonucleus scattering process is smaller than the inverse of the target nucleus radius, and the scattering amplitudes of the nucleons inside the nucleus can be added coherently, which leads to a large enhancement of the cross section. The observation of CE $\nu$ NS at COHERENT is consistent with the prediction of the Standard Model (SM), in which the CE $\nu$ NS process is generated through the weak neutral current [2]. Besides the active neutrinos through $Z$ boson exchange in the SM, any neutrino flavors including light right-handed $(\mathrm{RH})$ neutrinos can be produced in the final state of the $\mathrm{CE} \nu \mathrm{NS}$ process. Here the $\mathrm{RH}$ neutrinos refer to sterile neutrinos which do not carry any SM gauge charges. Since the production of the RH neutrinos will not violate the coherence condition if the momentum transfer are smaller than the inverse of the nucleus radius, the COHERENT observation thus provides us an opportunity to explore the new physics (NP) associated with general neutrino interactions in the presence of exotic fermion such as the $\mathrm{RH}$ neutrino.

Recently, different groups studied the conversion to an exotic fermion which could or could not be a dark matter (DM) particle ${ }^{1}$ in the coherent elastic neutrino-nucleus scattering $[3,6,8,9]$. The calculation of $\mathrm{CE} \nu \mathrm{NS}$ process depends on the specific interactions

\footnotetext{
${ }^{1}$ Note that the inverse process in which the exotic fermion as DM particle can be absorbed by the target and emit a neutrino could lead to distinct DM signal in DM direct detection. However, the decaying DM scenario usually faces the requirement of stability. Requiring the DM being stable at the Universe time scale would set a very stringent bound on the coupling and/or the DM mass. We will not interpret $\chi$ as a DM candidate and discuss the reverse process to detect this kind of DM scenario. We refer the discussion of the relevant neutrino-portal DM to refs. [3-7].
} 
between neutrino and SM quark sector. For instance, in the studies of neutrino interaction in ref. [6], the authors assumed that the interaction is mediated by a scalar field $a$ via the Yukawa couplings $g_{\chi} \bar{\chi} \nu a+g_{q} \bar{q} q a$. By contrast, if we consider the mediator $a$ as a pseudoscalar, the pseudoscalar quark current $\bar{q} i \gamma_{5} q a$ would lead to effective coupling for nuclear spin-dependent (SD) interaction which is determined by a sum over spin-up and spin-down nucleons with opposite signs [10]. Thus, this kind of contribution to CE $\nu$ NS is highly suppressed and usually neglected in the analysis of general neutrino interactions for heavy CsI nuclei in COHERENT experiment [1]. However, it is worth emphasizing that the tree-level interactions induced by pseudoscalar quark current can generate loop diagrams which in turn give scalar interactions and non-momentum-suppressed spin-independent (SI) scattering. The nuclear matrix element from these SI interactions could receive the enhancement of nuclear mass number in coherent scattering and compensate the suppression from the perturbative loop calculation. As a result, the full calculation involving the loop corrections leads to detectable observation for the pseudoscalar interaction. This loop effect has been taken into account in both simplified frameworks and UV complete models for detecting the Weakly Interacting Massive Particle (WIMP) in direct DM detection [11-27].

In this work we investigate both the tree-level and loop-level contributions to the $\mathrm{CE} \nu \mathrm{NS}$ in the framework of a simplified neutrino model. We assume generic neutrino currents interacting with an exotic fermion $\chi$ and SM quarks through a light spin-0 mediator $a$ with general $\mathrm{CP}$ phases. For the $\mathrm{CE} \nu \mathrm{NS}$ process, besides the scattering $\nu N \rightarrow \chi N$ with neutrinos converting to $\chi$ at tree-level, the loop diagrams can also induce elastic scattering $\nu N \rightarrow \nu N$ with the intermediate new fermion $\chi$ inside the loops. This additional contribution will affect the fit to COHERENT data and play an important role when the scattering process $\nu N \rightarrow \chi N$ is kinematically forbidden or the scalar current $\bar{q} q a$ is absent.

This paper is organized as follows. In section 2 we describe the simplified neutrino model. In section 3 we present the analytical expressions of the $\mathrm{CE} \nu \mathrm{NS}$ cross section. Both the tree-level and loop-level contributions are given in general forms. The numerical results are also shown. We discuss other relevant constraints on this model in section 4. Our conclusions are drawn in section 5 . Some calculational details are collected in the appendix.

\section{Simplified neutrino model with an exotic fermion}

We consider a Dirac fermion $\chi$ charged under lepton number being a SM gauge singlet. It is generally viewed as the sterile neutrino but can be a generic singlet fermion which mixes with the neutrino fields $\nu$. In the simplified neutrino model, the neutrinos interact with the new fermion $\chi$ through a spin-0 field $a$ and the mediator $a$ couples to the SM quarks as

$$
\mathcal{L} \supset g_{\chi} a \bar{\chi}\left(\cos \theta_{\chi}+i \gamma_{5} \sin \theta_{\chi}\right) \nu+\sum_{q} g_{q} a \bar{q}\left(\cos \theta_{q}+i \gamma_{5} \sin \theta_{q}\right) q+\text { h.c. }
$$

where $\theta_{\chi}$ denotes the relative CP phase angle between $\nu$ and $\chi$, and $\theta_{q}$ is the CP phase in quark sector. Here we use a universal coupling $g_{q}$ for the interaction between SM quarks and the mediator $a$. In practice, below we perform a generic hypothesis with different $g_{q}$ couplings for up-type and down-type quarks. This hypothesis can be realized in the 
two-Higgs-doublet model (2HDM) $[17,18,28]$ and one will see that the flavor constraints can be relaxed in this choice. The choice of $\left(\theta_{\chi}, \theta_{q}\right)=(0,0)$ is exactly the case with pure scalar mediator studied in ref. [6]. The other CP conserving case is $\left(\theta_{\chi}, \theta_{q}\right)=(\pi / 2, \pi / 2)$ with the field $a$ being a pseudoscalar mediator. Generally, the scenario with $\theta_{q}=\pi / 2$ leads to suppressed SD interaction at tree-level and the detectable signals are absent in neutrino experiments.

One should note that the above simplified hypothesis does not respect gauge invariance prior to the SM electroweak symmetry breaking. Thus, we expect that there exist additional couplings between $a$ and the SM Higgs in specific UV complete models [17]. Without loss of generality, we introduce a scalar trilinear coupling

$$
\mathcal{L} \supset \frac{1}{2} \lambda_{h a a} v_{0} h a^{2}
$$

where $v_{0}=\left(\sqrt{2} G_{F}\right)^{-1 / 2} \simeq 246 \mathrm{GeV}$ is the SM Higgs vacuum expectation value (vev). We will show that this interaction induces additional loop diagram in the scattering process.

In figure 1 we show the diagrams for the processes of neutrino interacting with the nucleus target at quark-level. For incoming neutrino processes, $A=\nu, B=\chi$. The treelevel diagram in figure 1 (a) is generated by two vertexes from the two couplings in the Lagrangian given in eq. (2.1) and a mediator $a$ in t channel. Figure 1 (b) is a triangle diagram intermediated by the $\lambda_{\text {haa }}$ coupling and the SM Higgs field $h$. Figures 1 (c) and (d) are the box diagrams formed by two $a$ fields in the internal lines. Recent developments in the loop calculation include the contributions from two-loop scattering diagrams in figures 1 (e) and (f) for scalar-type gluon operator $\frac{\alpha_{s}}{\pi} G_{\mu \nu}^{a} G^{a \mu \nu}[17,19,21]$. The full twoloop calculation can be obtained by integrating out the heavy quarks and the mediators in the loops. In next sections we will display how the effective operators for the neutrino scattering are formed from these diagrams.

\section{Coherent elastic neutrino-nucleus scattering}

For the incoming neutrino scattering $\nu\left(p_{1}\right) q\left(k_{1}\right) \rightarrow \chi\left(p_{2}\right) q\left(k_{2}\right)$ in figure 1 (a) with $A=$ $\nu, B=\chi$, we obtain the tree-level matrix element

$$
i M_{\text {tree }}=\sum_{q=a l l} \frac{-i}{t-m_{a}^{2}} g_{\chi} g_{q} \bar{\chi}\left(p_{2}\right)\left(c_{\theta_{\chi}}+i \gamma_{5} s_{\theta_{\chi}}\right) P_{L} \nu\left(p_{1}\right)\left[c_{\theta_{q}} \bar{q}\left(k_{2}\right) q\left(k_{1}\right)+s_{\theta_{q}} \bar{q}\left(k_{2}\right) i \gamma_{5} q\left(k_{1}\right)\right],
$$

where $P_{L}=\frac{1-\gamma_{5}}{2}$, the Mandelstam variable $t=\left(p_{1}-p_{2}\right)^{2}$ and $c_{x} \equiv \cos (x), s_{x} \equiv \sin (x)$. When $\left(\theta_{\chi}, \theta_{q}\right)=(0,0)$, this is exactly the case in ref. [6] with scalar mediator. In the case of $\theta_{q}=\pi / 2$, the SI contribution from tree-level diagram to the neutrino-nucleus scattering is zero.

For the elastic scattering process $\nu\left(p_{1}\right) q\left(k_{1}\right) \rightarrow \nu\left(p_{2}\right) q\left(k_{2}\right)$, there are multiple loop-level contributions as shown in figure 1 . We first consider the one-loop triangle diagram with SM Higgs exchange and the matrix element is

$$
\begin{aligned}
i M_{\text {triangle }}= & \sum_{q=\text { all }} \frac{-i \lambda_{h a a} v_{0} m_{\chi} g_{\chi}^{2}}{(4 \pi)^{2}\left(t-m_{h}^{2}\right)} C_{0}\left[p_{1}^{2},\left(p_{1}-p_{2}\right)^{2}, p_{2}^{2} ; m_{\chi}^{2}, m_{a}^{2}, m_{a}^{2}\right] \\
& \times \bar{\nu}\left(p_{2}\right)\left(c_{2 \theta_{\chi}}+i \gamma_{5} s_{2 \theta_{\chi}}\right) P_{L} \nu\left(p_{1}\right) \bar{q}\left(k_{2}\right) q\left(k_{1}\right) .
\end{aligned}
$$




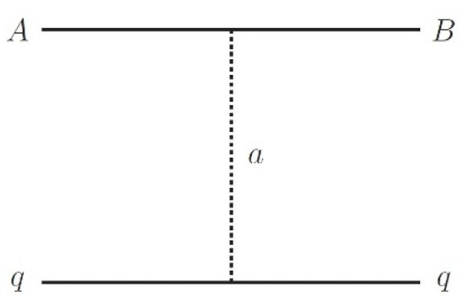

(a)

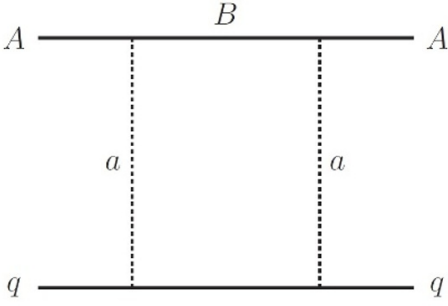

(c)

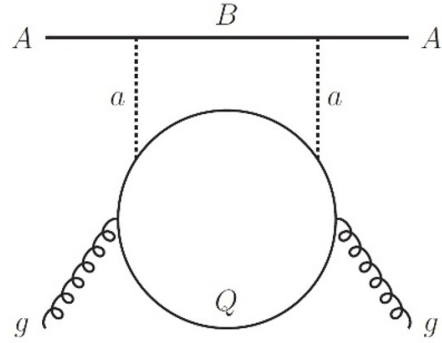

(e)

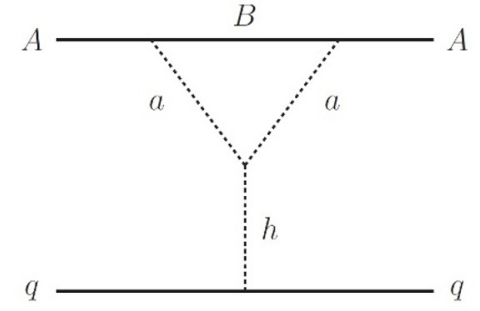

(b)

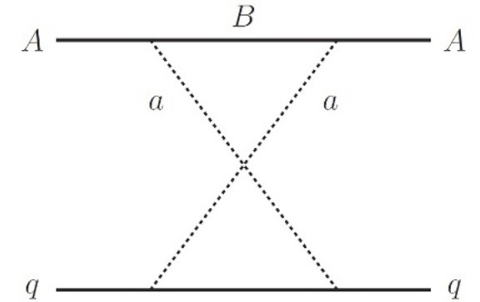

(d)

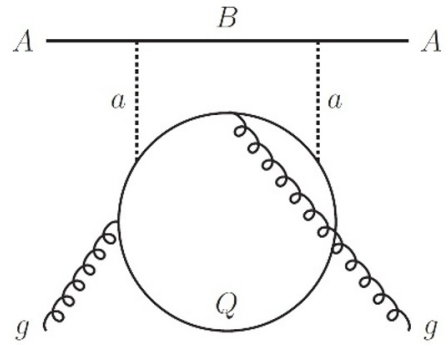

(f)

Figure 1. Diagrams for the processes of neutrino interacting with quark or gluon. For incoming neutrino processes, $A=\nu, B=\chi$.

In the zero momentum transfer limit $t \rightarrow 0$ and taking massless neutrinos, the PassarinoVeltman function $C_{0}$ can be simplified as shown in the appendix. One can see that the quark current is pure scalar-type in this diagram due to the SM Higgs exchange. The matrix element of the one-loop box diagrams is

$$
\begin{aligned}
i M_{\mathrm{box}}= & \sum_{q=u, d, s} \frac{i}{(4 \pi)^{2}} g_{\chi}^{2} g_{q}^{2} \frac{4 m_{\chi} m_{q}}{m_{a}^{2}} D_{00} \bar{\nu}\left(p_{2}\right)\left(c_{2 \theta_{\chi}}+i \gamma_{5} s_{2 \theta_{\chi}}\right) P_{L} \nu\left(p_{1}\right) \bar{q}\left(k_{2}\right) q\left(k_{1}\right) \\
& +\sum_{q=u, d, s} \frac{i}{(4 \pi)^{2}} g_{\chi}^{2} g_{q}^{2} 4 m_{\chi} m_{q} c_{\theta_{q}} D_{0} \bar{\nu}\left(p_{2}\right)\left(c_{2 \theta_{\chi}}+i \gamma_{5} s_{2 \theta_{\chi}}\right) P_{L} \nu\left(p_{1}\right) \bar{q}\left(k_{2}\right)\left(c_{\theta_{q}}+i \gamma_{5} s_{\theta_{q}}\right) q\left(k_{1}\right) \\
& +\sum_{q=u, d, s, c, b} \frac{i}{(4 \pi)^{2}} g_{\chi}^{2} g_{q}^{2} \frac{8}{m_{a}^{2}} D_{001} \bar{\nu}\left(p_{2}\right) i \partial^{\mu} \gamma^{\nu} P_{L} \nu\left(p_{1}\right) O_{\mu \nu}^{q} \\
& +\sum_{q=u, d, s, c, b} \frac{i}{(4 \pi)^{2}} g_{\chi}^{2} g_{q}^{2} \frac{4 m_{\chi}}{m_{a}^{2}} D_{11} \bar{\nu}\left(p_{2}\right)\left(c_{2 \theta_{\chi}}+i \gamma_{5} s_{2 \theta_{\chi}}\right) i \partial^{\mu} i \partial^{\nu} P_{L} \nu\left(p_{1}\right) O_{\mu \nu}^{q}
\end{aligned}
$$

where $O_{\mu \nu}^{q}$ is the twist-2 operator for quark

$$
O_{\mu \nu}^{q}=\frac{i}{2} \bar{q}\left(\partial_{\mu} \gamma_{\nu}+\partial_{\nu} \gamma_{\mu}-\frac{1}{2} g_{\mu \nu} \not \partial\right) q .
$$

The Passarino-Veltman functions are also collected in the appendix. 
For the heavy quark loops in the two-loop diagrams, we calculate the amplitude using the Fock-Schwinger gauge for the gluon background field [12, 29]. First, the amplitude contributing to the effective operator $a a G_{\mu \nu}^{a} G^{a \mu \nu}$ is

$$
i M_{a a G G}=i \Pi_{G}\left(\ell^{2}\right) \frac{\alpha_{s}}{12 \pi} G_{\rho \sigma}^{a} G^{a \rho \sigma}+i \Pi_{\tilde{G}}\left(\ell^{2}\right) \frac{\alpha_{s}}{8 \pi} G_{\rho \sigma}^{a} \tilde{G}^{a \rho \sigma},
$$

where $G^{a \mu \nu}$ is the gluon field strength tensor and $\tilde{G}^{a \mu \nu}=\frac{1}{2} \epsilon^{\mu \nu \alpha \beta} G_{\alpha \beta}^{a}$. Then, the complete two-loop matrix element in figures 1 (e) and (f) reads

$$
\begin{aligned}
i M_{2-\text { loop }}= & -g_{\chi}^{2} \int \frac{d^{4} \ell}{(2 \pi)^{4}} \bar{\nu}\left(p_{2}\right)\left[\ell+m_{\chi}\left(c_{2 \theta_{\chi}}+i \gamma_{5} s_{2 \theta_{\chi}}\right)\right] P_{L} \nu\left(p_{1}\right) \\
& \times \frac{1}{\left[\left(\ell+p_{1}\right)^{2}-m_{\chi}^{2}\right]\left(\ell^{2}-m_{a}^{2}\right)^{2}}\left[\Pi_{G}\left(\ell^{2}\right) \frac{\alpha_{s}}{12 \pi} G_{\rho \sigma}^{a} G^{a \rho \sigma}+\Pi_{\tilde{G}}\left(\ell^{2}\right) \frac{\alpha_{s}}{8 \pi} G_{\rho \sigma}^{a} \tilde{G}^{a \rho \sigma}\right] \\
= & {\left[C_{G, S} \bar{\nu}\left(p_{2}\right) P_{L} \nu\left(p_{1}\right)+C_{G, P S} \bar{\nu}\left(p_{2}\right) i \gamma_{5} P_{L} \nu\left(p_{1}\right)\right] \frac{-\alpha_{s}}{12 \pi} G_{\rho \sigma}^{a} G^{a \rho \sigma} } \\
& +\left[C_{\tilde{G}, S} \bar{\nu}\left(p_{2}\right) P_{L} \nu\left(p_{1}\right)+C_{\tilde{G}, P S} \bar{\nu}\left(p_{2}\right) i \gamma_{5} P_{L} \nu\left(p_{1}\right)\right] \frac{\alpha_{s}}{8 \pi} G_{\rho \sigma}^{a} \tilde{G}^{a \rho \sigma}
\end{aligned}
$$

where $\ell$ denotes the momentum of the mediator $a$ and

$$
\begin{aligned}
C_{G, S} & =\frac{i}{(4 \pi)^{2}} \sum_{Q=c, b, t} g_{\chi}^{2} g_{Q}^{2} m_{\chi} c_{2 \theta_{\chi}} F_{G}\left(p_{1}^{2}, m_{\chi}^{2}, m_{a}^{2}, m_{Q}^{2}\right), \\
C_{G, P S} & =\frac{i}{(4 \pi)^{2}} \sum_{Q=c, b, t} g_{\chi}^{2} g_{Q}^{2} m_{\chi} s_{2 \theta_{\chi}} F_{G}\left(p_{1}^{2}, m_{\chi}^{2}, m_{a}^{2}, m_{Q}^{2}\right), \\
C_{\tilde{G}, S} & =-\frac{i}{(4 \pi)^{2}} \sum_{Q=c, b, t} g_{\chi}^{2} g_{Q}^{2} m_{\chi} c_{2 \theta_{\chi}} F_{\tilde{G}}\left(p_{1}^{2}, m_{\chi}^{2}, m_{a}^{2}, m_{Q}^{2}\right), \\
C_{\tilde{G}, P S} & =-\frac{i}{(4 \pi)^{2}} \sum_{Q=c, b, t} g_{\chi}^{2} g_{Q}^{2} m_{\chi} s_{2 \theta_{\chi}} F_{\tilde{G}}\left(p_{1}^{2}, m_{\chi}^{2}, m_{a}^{2}, m_{Q}^{2}\right) .
\end{aligned}
$$

The above $\Pi_{G}\left(\ell^{2}\right), \Pi_{\tilde{G}}\left(\ell^{2}\right)$ and $F_{G}, F_{\tilde{G}}$ functions are all given in the appendix.

The nucleon form factors are defined as $[30,31]$

$$
\begin{aligned}
\left\langle N\left|m_{q} \bar{q} q\right| N\right\rangle & =m_{N} f_{q}^{N} \bar{N} N, & q & =u, d, s, \\
\left\langle N\left|m_{Q} \bar{Q} Q\right| N\right\rangle & =\left\langle N\left|\frac{-\alpha_{s}}{12 \pi} G_{\mu \nu}^{a} G^{a \mu \nu}\right| N\right\rangle=\frac{2}{27} m_{N} f_{G}^{N} \bar{N} N, & Q & =c, b, t, \\
\left\langle N\left|O_{\mu \nu}^{q}\right| N\right\rangle & =\frac{1}{m_{N}}\left(p_{\mu}^{N} p_{\nu}^{N}-\frac{1}{4} m_{N}^{2} g_{\mu \nu}\right)\left(q^{N}(2)+\bar{q}^{N}(2)\right) \bar{N} N, & & =u, d, s, c, b,
\end{aligned}
$$

for the SI interactions and those for SD interactions are

$$
\begin{aligned}
\left\langle N\left|m_{q} \bar{q} i \gamma_{5} q\right| N\right\rangle & =F_{P}^{q / N}\left(q^{2}\right) \bar{N} i \gamma_{5} N, \quad q=u, d, s \\
\left\langle N\left|m_{Q} \bar{Q} i \gamma_{5} Q\right| N\right\rangle & =\left\langle N\left|\frac{\alpha_{s}}{8 \pi} G_{\mu \nu}^{a} \tilde{G}^{a \mu \nu}\right| N\right\rangle=F_{\tilde{G}}^{N}\left(q^{2}\right) \bar{N} i \gamma_{5} N, \quad Q=c, b, t .
\end{aligned}
$$


Next, we can obtain the matrix elements at nucleon-level

$$
\begin{aligned}
i M_{\text {tree }}^{N}= & \frac{-i}{t-m_{a}^{2}} g_{\chi} g_{q} c_{\theta_{q}}\left(\sum_{q=u, d, s} \frac{m_{N}}{m_{q}} f_{q}^{N}+\sum_{Q=c, b, t} \frac{2}{27} \frac{m_{N}}{m_{Q}} f_{G}^{N}\right) \\
& \times \bar{\chi}\left(p_{2}\right)\left(c_{\theta_{\chi}}+i \gamma_{5} s_{\theta_{\chi}}\right) P_{L} \nu\left(p_{1}\right) \bar{N}\left(k_{2}\right) N\left(k_{1}\right)+\mathrm{SD} \\
i M_{\text {triangle }}^{N}= & \frac{-i \lambda_{h a a} v_{0} m_{\chi} g_{\chi}^{2}}{(4 \pi)^{2}\left(t-m_{h}^{2}\right)} C_{0}\left(\sum_{q=u, d, s} \frac{m_{N}}{m_{q}} f_{q}^{N}+\sum_{Q=c, b, t} \frac{2}{27} \frac{m_{N}}{m_{Q}} f_{G}^{N}\right) \\
& \times \bar{\nu}\left(p_{2}\right)\left(c_{2 \theta_{\chi}}+i \gamma_{5} s_{2 \theta_{\chi}}\right) P_{L} \nu\left(p_{1}\right) \bar{N}\left(k_{2}\right) N\left(k_{1}\right), \\
i M_{\mathrm{box}}^{N}= & \sum_{q=u, d, s} \frac{i}{(4 \pi)^{2}} g_{\chi}^{2} g_{q}^{2} f_{q}^{N}\left(\frac{4 m_{\chi} m_{N}}{m_{a}^{2}} D_{00}+4 m_{\chi} m_{N} c_{\theta_{q}}^{2} D_{0}\right) \\
& \times \bar{\nu}\left(p_{2}\right)\left(c_{2 \theta_{\chi}}+i \gamma_{5} s_{2 \theta_{\chi}}\right) P_{L} \nu\left(p_{1}\right) \bar{N}\left(k_{2}\right) N\left(k_{1}\right)+\mathrm{SD}, \\
i M_{2-\text { loop }}^{N}= & {\left[C_{G, S} \bar{\nu}\left(p_{2}\right) P_{L} \nu\left(p_{1}\right)+C_{G, P S} \bar{\nu}\left(p_{2}\right) i \gamma_{5} P_{L} \nu\left(p_{1}\right)\right] \frac{2}{27} m_{N} f_{G}^{N} \bar{N}\left(k_{2}\right) N\left(k_{1}\right)+\mathrm{SD} . }
\end{aligned}
$$

Here SD stands for SD terms which will be omitted in the following calculation.

Since $\chi$ is not detected in a neutrino scattering experiment, the total differential cross section of $\mathrm{CE} \nu \mathrm{NS}$ can be written as

$$
\frac{d \sigma}{d T}=\frac{d \sigma_{\mathrm{SM}}}{d T}+\frac{d \sigma_{\text {tree }}}{d T}+\frac{d \sigma_{\mathrm{loop}}}{d T}
$$

where $T$ is the nuclear recoil energy. The SM differential cross section is given by

$$
\frac{d \sigma_{\mathrm{SM}}}{d T}=\frac{G_{F}^{2} M}{2 \pi}\left[Z g_{p}^{V}+N g_{n}^{V}\right]^{2} F^{2}\left(Q^{2}\right)\left(2-\frac{M T}{E^{2}}\right),
$$

where $F\left(Q^{2}\right)$ refers to the nuclear form factor with the moment transfer $Q^{2}=2 M T$. We take the Helm parameterization [32] for the nuclear form factor. Note that employing a different form factor parameterization has a negligible effect on the COHERENT spectrum [33, 34], and the form factor uncertainty driven by the nucleon density distribution has been taken into account in our analysis. Here $M$ is the mass of target nucleus, $E$ is the incoming neutrino energy, $Z(N)$ is the number of protons (neutrons) in the target nucleus, $g_{n}^{V}=-\frac{1}{2}$ and $g_{p}^{V}=\frac{1}{2}-2 \sin ^{2} \theta_{W}$ are the SM weak couplings with $\theta_{W}$ being the weak mixing angle.

From eq. (3.16), the tree-level differential cross section of $\nu N \rightarrow \chi N$ is

$$
\frac{d \sigma_{\text {tree }}}{d T}=\frac{g_{\chi}^{2} g_{N}^{2} F^{2}\left(Q^{2}\right)}{16 \pi E^{2}\left(m_{a}^{2}+2 M T\right)^{2}}(2 M+T)\left(2 M T+m_{\chi}^{2}\right)
$$

where

$$
g_{N}=Z m_{p}\left(\sum_{q=u, d, s} \frac{f_{q}^{p}}{m_{q}}+\sum_{Q=c, b, t} \frac{2}{27} \frac{f_{G}^{p}}{m_{Q}}\right) g_{q} c_{\theta_{q}}+N m_{n}\left(\sum_{q=u, d, s} \frac{f_{q}^{n}}{m_{q}}+\sum_{Q=c, b, t} \frac{2}{27} \frac{f_{G}^{n}}{m_{Q}}\right) g_{q} c_{\theta_{q}} .
$$


Note that in order to produce a massive fermion $\chi$ in the scattering $\nu N \rightarrow \chi N$, the energy of the incident neutrinos should be larger than a minimal energy $[3,9]$, i.e.,

$$
E>m_{\chi}+\frac{m_{\chi}^{2}}{2 M}
$$

From eqs. (3.17), (3.18) and (3.19), we can write the loop-level differential cross section as

$$
\frac{d \sigma_{\text {loop }}}{d T}=\frac{G_{\text {loop }}^{2} M}{8 \pi E^{2}} F^{2}\left(Q^{2}\right)\left(2 M T+T^{2}\right)
$$

where

$$
\begin{aligned}
G_{\text {loop }}= & \frac{Z}{(4 \pi)^{2}} g_{\chi}^{2} m_{\chi} m_{p}\left[\frac{\lambda_{h a a} v_{0}}{2 M T+m_{h}^{2}}\left(\sum_{q=u, d, s} \frac{f_{q}^{p}}{m_{q}}+\sum_{Q=c, b, t} \frac{2}{27} \frac{f_{G}^{p}}{m_{Q}}\right) C_{0}\right. \\
& \left.+4 \sum_{q=u, d, s} g_{q}^{2} f_{q}^{p}\left(\frac{D_{00}}{m_{a}^{2}}+c_{\theta_{q}}^{2} D_{0}\right)+\frac{2}{27} f_{G}^{p} \sum_{Q=c, b, t} g_{Q}^{2} F_{G}\left(p_{1}^{2}, m_{\chi}^{2}, m_{a}^{2}, m_{Q}^{2}\right)\right] \\
& +\frac{N}{(4 \pi)^{2}} g_{\chi}^{2} m_{\chi} m_{n}\left[\frac{\lambda_{h a a} v_{0}}{2 M T+m_{h}^{2}}\left(\sum_{q=u, d, s} \frac{f_{q}^{n}}{m_{q}}+\sum_{Q=c, b, t} \frac{2}{27} \frac{f_{G}^{n}}{m_{Q}}\right) C_{0}\right. \\
& \left.+4 \sum_{q=u, d, s} g_{q}^{2} f_{q}^{n}\left(\frac{D_{00}}{m_{a}^{2}}+c_{\theta_{q}}^{2} D_{0}\right)+\frac{2}{27} f_{G}^{n} \sum_{Q=c, b, t} g_{Q}^{2} F_{G}\left(p_{1}^{2}, m_{\chi}^{2}, m_{a}^{2}, m_{Q}^{2}\right)\right] .
\end{aligned}
$$

One can see that the total differential cross section has no dependence on the mixing angle $\theta_{\chi}$. This is because the tree-level amplitude and the loop-level amplitude have no interference in this case and the neutrinos in the external legs are nearly massless. The measurement of the SM Higgs decay at the LHC [35] implies the constraint on the coupling $\lambda_{h a a} \lesssim 0.01$ [21]. In addition, the triangle diagram is suppressed by a factor of $m_{\chi} v_{0} / m_{h}^{2} \sim$ $\mathcal{O}\left(10^{-5}\right)$ for $m_{\chi} \sim \mathcal{O}(1) \mathrm{MeV}$. The flavor physics also sets stringent constraints on the coupling $g_{q}$ for up-type quarks as discussed below. Thus, in the numerical calculation, we neglect the coupling for up-type quarks and the triangle diagram and the two-loop diagrams dominated by top quark contribution. We checked that this ignorance does not affect our conclusion. For the nucleon form factors in SI interactions, we adopt the default values in micrOMEGAs [36, 37].

The CE $\nu$ NS process has been recently observed by the COHERENT experiment in a low-threshold CsI detector at the 6.7 $\sigma$ CL. The neutrinos measured at COHERENT are generated from the stopped pion decays and the muon decays, and their fluxes are well known and given by

$$
\begin{aligned}
\phi_{\nu_{\mu}}\left(E_{\nu_{\mu}}\right) & =\mathcal{N}_{0} \frac{2 m_{\pi}}{m_{\pi}^{2}-m_{\mu}^{2}} \delta\left(1-\frac{2 E_{\nu_{\mu}} m_{\pi}}{m_{\pi}^{2}-m_{\mu}^{2}}\right), \\
\phi_{\nu_{e}}\left(E_{\nu_{e}}\right) & =\mathcal{N}_{0} \frac{192}{m_{\mu}}\left(\frac{E_{\nu_{e}}}{m_{\mu}}\right)^{2}\left(\frac{1}{2}-\frac{E_{\nu_{e}}}{m_{\mu}}\right) \\
\phi_{\bar{\nu}_{\mu}}\left(E_{\bar{\nu}_{\mu}}\right) & =\mathcal{N}_{0} \frac{64}{m_{\mu}}\left(\frac{E_{\bar{\nu}_{\mu}}}{m_{\mu}}\right)^{2}\left(\frac{3}{4}-\frac{E_{\bar{\nu}_{\mu}}}{m_{\mu}}\right)
\end{aligned}
$$




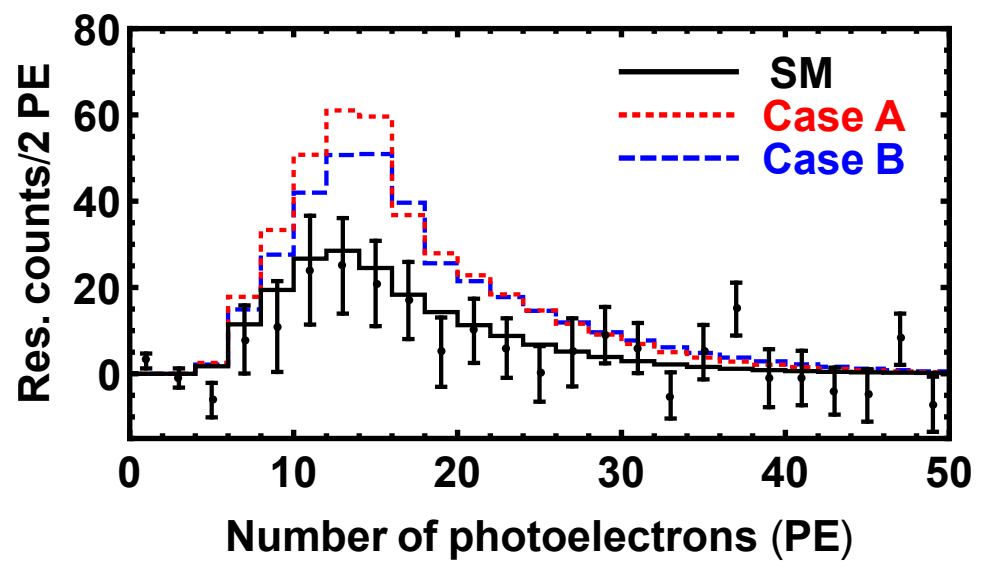

Figure 2. The expected $\mathrm{CE} \nu \mathrm{NS}$ residual event as a function of the number of photoelectrons at COHERENT. The black solid lines correspond to the SM case, and the red dotted (blue dashed) lines correspond to Case A (B) with $\theta_{q}=0, m_{\chi}=10 \mathrm{MeV}, m_{a}=100 \mathrm{MeV}$, and $g_{\chi} g_{q}=5.0 \times 10^{-9}$ $\left(\theta_{q}=\pi / 2, m_{\chi}=100 \mathrm{MeV}, m_{a}=200 \mathrm{MeV}\right.$, and $\left.g_{\chi} g_{q}=0.01\right)$.

where $\mathcal{N}_{0}$ is a normalization factor determined by the setup of the COHERENT experiment. The $\nu_{\mu}$ component is produced from the stopped pion decays, $\pi^{+} \rightarrow \mu^{+}+\nu_{\mu}$, which yield a monoenergetic flux at $\left(m_{\pi}^{2}-m_{\mu}^{2}\right) /\left(2 m_{\pi}\right) \simeq 30 \mathrm{MeV}$. The $\bar{\nu}_{\mu}$ and $\nu_{e}$ components are produced from the subsequent muon decays, $\mu^{+} \rightarrow e^{+}+\bar{\nu}_{\mu}+\nu_{e}$, and their energies have a kinematic upper bound at $m_{\mu} / 2 \simeq 53 \mathrm{MeV}$.

The presence of $\chi$-neutrino interaction will modify the COHERENT spectrum, which can be seen in figure 2. We select two benchmark points to illustrate the effects of modified spectra:

- Case A: $\theta_{q}=0, m_{\chi}=10 \mathrm{MeV}, m_{a}=100 \mathrm{MeV}$, and $g_{\chi} g_{q}=5.0 \times 10^{-9}$,

- Case B: $\theta_{q}=\pi / 2, m_{\chi}=100 \mathrm{MeV}, m_{a}=200 \mathrm{MeV}$, and $g_{\chi} g_{q}=0.01$.

In Case A, the modification to the SM spectrum is dominated by the tree-level scattering process, $\nu N \rightarrow \chi N$, and the loop-level contribution is negligible due to the small coupling constants. In Case $\mathrm{B}$, since $m_{\chi} \gtrsim 53 \mathrm{MeV}$, the tree-level process is kinematically forbidden, and the modification to the SM spectrum is only contributed by the loop-level diagrams.

Following ref. [9], we evaluate the statistical significance of BSM by defining

$$
\chi^{2}=\sum_{i=4}^{15}\left[\frac{N_{\text {meas }}^{i}-N_{\text {th }}^{i}(1+\alpha)-B_{\text {on }}(1+\beta)}{\sigma_{\text {stat }}^{i}}\right]^{2}+\left(\frac{\alpha}{\sigma_{\alpha}}\right)^{2}+\left(\frac{\beta}{\sigma_{\beta}}\right)^{2},
$$

where $N_{\text {meas }}^{i}$ and $N_{\text {th }}^{i}$ denotes the number of measured (predicted) events per energy bin, respectively. $\alpha(\beta)$ represents the nuisance parameters for the signal rate (the beamon background) with a uncertainty of $\sigma_{\alpha}=0.28\left(\sigma_{\beta}=0.25\right)$ [1]. The neutrino flux uncertainty (10\%), form factor uncertainty (5\%), signal acceptance uncertainty $(5 \%)$, and quenching factor uncertainty $(25 \%)$ are included in the signal rate uncertainty $\sigma_{\alpha}$. The statistical uncertainty per energy bin is calculated by $\sigma_{\text {stat }}^{i}=\sqrt{N_{\text {meas }}^{i}+2 B_{\mathrm{SS}}^{i}+B_{\mathrm{on}}^{i}}$ with 

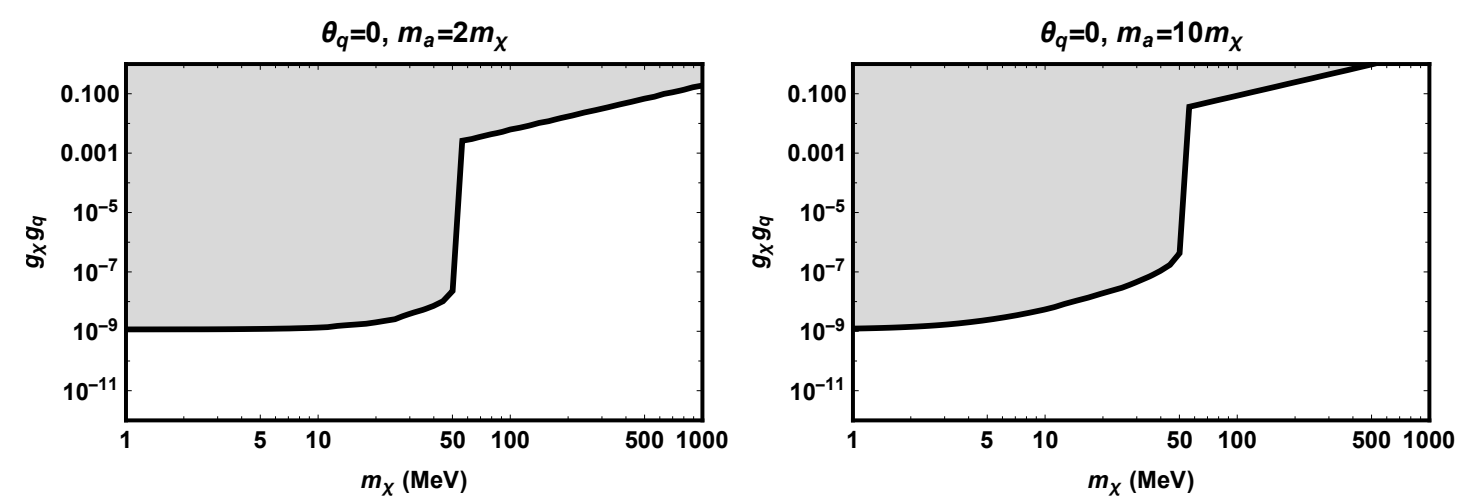

Figure 3. The 90\% CL upper bounds on $g_{\chi} g_{q}$ as a function of the mass $m_{\chi}$ from COHERENT. We assume $\theta_{q}=0$ and $m_{a}=2 m_{\chi}$ (left), or $m_{a}=10 m_{\chi}$ (right).

$B_{\mathrm{SS}}$ being the steady-state background from the anti-coincident data, and $B_{\mathrm{on}}$ the beam-on background mainly consists of prompt neutrons.

To obtain the bounds on the simplified neutrino model, we first set $\theta_{q}=0$ and $m_{a}=$ $2 m_{\chi}$ or $10 m_{\chi}$, and scan over possible values of the product of the coefficients $g_{\chi} g_{q}$ for a given $m_{\chi}$. The $90 \%$ CL upper bounds on $g_{\chi} g_{q}$ as a function of $m_{\chi}$ are shown in figure 3 . As we see from figure 3 , for $m_{\chi} \lesssim 53 \mathrm{MeV}$, the upper bounds on $g_{\chi} g_{q}$ are very strong, and can reach as small as $10^{-9}$ for $m_{\chi}=1 \mathrm{MeV}$. For $m_{\chi} \gtrsim 53 \mathrm{MeV}$, however, the tree-level process $\nu N \rightarrow \chi N$ is kinematically forbidden and the bounds become much weaker since the contribution from the loop diagrams is relatively small. Thus, there exhibits a kink around $m_{\chi} \simeq 53 \mathrm{MeV}$. Compare the left panel of figure 3 to the right panel, we see that in general the bounds become weaker as the mediator mass increases.

We also fix $\theta_{q}=\pi / 2$ and obtain the $90 \%$ CL upper bounds on $g_{\chi} g_{q}$ as a function of $m_{\chi}$. The results are shown in the left and right panel of figure 4 for $m_{a}=2 m_{\chi}$ and $m_{a}=10 m_{\chi}$, respectively. From eq. (3.23), we see that for $\theta_{q}=\pi / 2$, the SI terms from the tree-level process vanish, and the bounds are mainly determined by the loop-level contribution. ${ }^{2}$ For $m_{\chi} \simeq 53 \mathrm{MeV}$, the pure loop-level contribution constrains $g_{\chi} g_{q}$ to be smaller than 0.003 (0.06) for $m_{a}=2 m_{\chi}\left(10 m_{\chi}\right)$.

\section{Other constraints}

\subsection{Flavor constraint}

The simplified neutrino model receives constraints from the invisible rare decays such as $K^{+} \rightarrow \pi^{+}+$invisible via flavor changing neutral currents [38]. This rare decay is recently measured by the NA62 experiment at CERN [39]. In this model the partial width for

\footnotetext{
${ }^{2}$ In principle, for $m_{\chi} \lesssim 53 \mathrm{MeV}$, the $\mathrm{SD}$ terms also contribute to the $\mathrm{CE} \nu \mathrm{NS}$ process. The contribution is nonzero for the odd-even nucleus in the CsI detector. As it is highly suppressed compared with the SI terms, we do not consider its contribution here.
} 

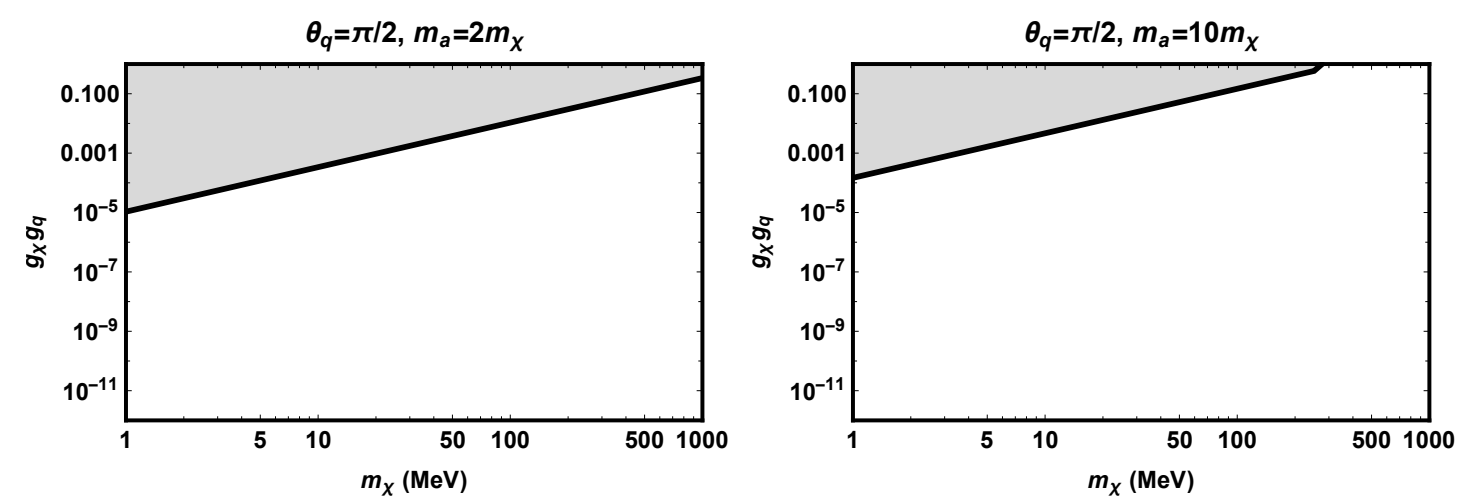

Figure 4. Same as figure 3, except for $\theta_{q}=\pi / 2$.

$K^{+} \rightarrow \pi^{+} a$ is $[40,41]$

$$
\begin{aligned}
\Gamma\left(K^{+} \rightarrow \pi^{+} a\right) & =\frac{\left|f\left(g_{q}\right)\right|^{2}+\left|k\left(g_{q}\right)\right|^{2}}{16 \pi m_{K^{+}}^{3}} \lambda^{1 / 2}\left(m_{K^{+}}^{2}, m_{\pi^{+}}^{2}, m_{a}^{2}\right), \\
f\left(g_{q}\right) & =\frac{3 m_{K^{+}}^{2}}{32 \pi^{2} v_{0}^{2} m_{s}} g_{q} \cos \theta_{q} f_{+}(0) \sum_{q=u, c, t} m_{q}^{2} V_{q d}^{*} V_{q s}, \\
k\left(g_{q}\right) & =\frac{4 m_{K^{+}}^{2}}{32 \pi^{2} v_{0}^{2} m_{s}} g_{q} \sin \theta_{q} f_{+}(0) \sum_{q=u, c, t} m_{q}^{2} V_{q d}^{*} V_{q s} \ln \left(\frac{m_{W}^{2}}{m_{q}^{2}}\right),
\end{aligned}
$$

where $\lambda(x, y, z)=x^{2}+y^{2}+z^{2}-2 x y-2 y z-2 x z$ and $f_{+}(0) \approx 0.9709$ is the vector form factor at zero momentum transfer. For $K_{L} \rightarrow \pi^{0}$ decay, one needs to replace $V_{q d}^{*} V_{q s}$ by $\operatorname{Re}\left[V_{q d}^{*} V_{q s}\right]$ and change the masses of kaon and pion. One can see that the contribution is from the up-type quarks in the loop and ref. [6] estimated the bound as $g_{q} \lesssim 1.58 \times 10^{-4}$. Note that the above result is on the analogy of the derivations for the SM-like Higgs in ref. [41] and the CP-odd Higgs in the two Higgs doublet model in ref. [40]. The actual calculation would suffer from a problem of UV divergence due to the fact that the simplified model here is not gauge-invariant $[15,38]$. The reliable estimate of the flavor observable relies on the UV completion realization. This problem does not affect our assumption of the couplings below and the corresponding conclusions.

The flavor observables through $K \rightarrow \pi$ transitions thus set stringent constraints on $g_{q}$ coupling for up-type quarks. We assumed non-universal $g_{q}$ couplings for up-type and down-type quarks and neglected the up-type quark coupling in the above calculations.

\subsection{LHC constraint}

If the new fermion $\chi$ is long-lived enough, the search for events with large missing transverse momentum with an energetic jet or the third generation SM quarks at the Large Hadron Collider (LHC) may place bounds on the coupling $g_{\chi} g_{q}$. One can see that there is no severe bound on the very light long-lived particle of interest with spin-0 mediator from monojet search $[42,43]$ or the coupling of down-type quarks from the production in association with bottom quarks [44]. The most stringent limits are from the associated production of 
$t \bar{t}$ with missing transverse momentum. The pseudoscalar mediator mass around $20 \mathrm{GeV}$ is excluded at $95 \%$ confidence level, assuming the $\chi$ mass being $1 \mathrm{GeV}$ and unitary top couplings [45]. This constraint is not severe in our discussion with absent up-type quark coupling. In addition, as stated before, the search for Higgs invisible decay sets constraint on the coupling $\lambda_{h a a} \lesssim 0.01$ in a specific Higgs portal model $[35,46]$.

\subsection{Long-lived hypothesis}

Although we did not interpret the fermion $\chi$ as DM in the above discussion, here we briefly discuss the validation of our result on the new fermion $\chi$ if it is long-lived. In our simplified scenario, the two-body process $\chi \rightarrow \nu \gamma$ via a closed quark loop vanishes as its amplitude becomes

$$
M(\chi \rightarrow \nu \gamma) \propto \int \frac{d^{4} k}{(2 \pi)^{4}} \frac{k \cdot \epsilon(q)}{\left(k^{2}-m_{q}^{2}\right)\left[(k+q)^{2}-m_{q}^{2}\right]} \propto \epsilon \cdot q=0,
$$

where $\epsilon$ is the photon polarization vector, $k$ and $q$ denote the integral loop-momentum and the momentum of the external photon, respectively. The leading decay process is thus $\chi \rightarrow \nu \gamma \gamma$ with two photons radiated from the closed quark loop. According to ref. [7], the decay width depends on both $m_{\chi}$ and $\frac{g_{\chi} g_{q}}{m_{a}^{2}}$

$$
\Gamma(\chi \rightarrow \nu \gamma \gamma) \propto m_{\chi}^{7}\left(\frac{g_{\chi} g_{q}}{m_{a}^{2}}\right)^{4}
$$

Therefore, if $m_{\chi}$ is small enough, the fermion $\chi$ can be long-lived. As seen in figure 3 , the constraint from COHERENT is independent of $m_{\chi}$ in low mass region and valid for very light fermion $\chi$. We refer a UV complete model to ref. [3].

\section{Conclusions}

We investigate the constraint on general neutrino interactions with an exotic fermion in the coherent neutrino-nucleus scattering experiments. We consider both the tree-level and loop-level contributions to the $\mathrm{CE} \nu \mathrm{NS}$ process in the framework of a simplified neutrino model with a new Dirac fermion $\chi$ and a spin-0 mediator $a$. The couplings between the mediator and the new fermion $\chi$ (the SM quarks) is parameterized by $g_{\chi}\left(g_{q}\right)$. For the $\mathrm{CP}$ phase in the quark sector $\theta_{q}=0(\pi / 2)$, the detection processes are dominated by the tree-level (loop-level) contribution. We find that for $\theta_{q}=0$, the COHERENT experiment can set the upper bound of $g_{\chi} g_{q}$ as small as $10^{-9}$ for $m_{\chi}=1 \mathrm{MeV}$. Also, by including the loop-level contribution, the COHERENT data is also sensitive to the mass region with $m_{\chi} \gtrsim 53 \mathrm{MeV}$, which is the maximal energy of the incoming neutrinos measured at COHERENT. In general, the bounds become weaker as the mediator mass increases. In addition, when $\theta_{q}=\pi / 2$, the COHERENT spectrum can be also modified after taking the loop-level contribution into account. By fitting to the COHERENT data, we find that the loop-level contribution constrains $g_{\chi} g_{q}$ as small as $0.003(0.06)$ for $m_{\chi} \simeq 53 \mathrm{MeV}$ and $m_{a}=2 m_{\chi}\left(10 m_{\chi}\right)$. 


\section{Acknowledgments}

TL is supported by the National Natural Science Foundation of China (Grant No. 11975129, 12035008) and "the Fundamental Research Funds for the Central Universities", Nankai University (Grant No. 63196013). JL is supported by the National Natural Science Foundation of China (Grant No. 11905299), Guangdong Basic and Applied Basic Research Foundation (Grant No. 2020A1515011479), the Fundamental Research Funds for the Central Universities, and the Sun Yat-Sen University Science Foundation.

\section{A Loop diagram calculation in neutrino-nucleus scattering}

For the elastic scattering process $\nu\left(p_{1}\right) q\left(k_{1}\right) \rightarrow \nu\left(p_{2}\right) q\left(k_{2}\right)$, the Passarino-Veltman function for the triangle diagram is

$$
C_{0}\left[p_{1}^{2},\left(p_{1}-p_{2}\right)^{2}, p_{2}^{2} ; m_{\chi}^{2}, m_{a}^{2}, m_{a}^{2}\right]=C_{0}\left[0,0,0 ; m_{\chi}^{2}, m_{a}^{2}, m_{a}^{2}\right]=\frac{m_{\chi}^{2} \ln \left(\frac{m_{a}^{2}}{m_{\chi}^{2}}\right)-m_{a}^{2}+m_{\chi}^{2}}{\left(m_{a}^{2}-m_{\chi}^{2}\right)^{2}} .
$$

The Passarino-Veltman functions for the one-loop box diagrams are defined as

$$
\begin{aligned}
& D_{0}\left(p_{1}, m_{\chi}, m_{a}\right) \equiv D_{0}\left[p_{1}^{2}, p_{1}^{2}, 0,0,0, p_{1}^{2} ; 0, m_{\chi}^{2}, m_{a}^{2}, m_{a}^{2}\right]=\frac{-m_{a}^{2}+m_{\chi}^{2}+m_{a}^{2} \ln \left(\frac{m_{a}^{2}}{m_{\chi}^{2}}\right)}{m_{a}^{2}\left(m_{a}^{2}-m_{\chi}^{2}\right)^{2}}, \\
& D_{00}\left(p_{1}, m_{\chi}, m_{a}\right) \equiv D_{00}\left[p_{1}^{2}, p_{1}^{2}, 0,0,0, p_{1}^{2} ; 0, m_{\chi}^{2}, 0, m_{a}^{2}\right]-D_{00}\left[p_{1}^{2}, p_{1}^{2}, 0,0,0, p_{1}^{2} ; 0, m_{\chi}^{2}, m_{a}^{2}, m_{a}^{2}\right] \\
&=\frac{m_{a}^{2}-m_{\chi}^{2}-m_{a}^{2} \ln \left(\frac{m_{a}^{2}}{m_{\chi}^{2}}\right)}{4\left(m_{a}^{2}-m_{\chi}^{2}\right)^{2}}, \\
& D_{11}\left(p_{1}, m_{\chi}, m_{a}\right) \equiv D_{11}\left[p_{1}^{2}, p_{1}^{2}, 0,0,0, p_{1}^{2} ; 0, m_{\chi}^{2}, 0, m_{a}^{2}\right]-D_{11}\left[p_{1}^{2}, p_{1}^{2}, 0,0,0, p_{1}^{2} ; 0, m_{\chi}^{2}, m_{a}^{2}, m_{a}^{2}\right] \\
&=m_{a}^{2} \frac{\left(m_{a}^{2}-m_{\chi}^{2}\right)\left(m_{a}^{2}+5 m_{\chi}^{2}\right)-2 m_{\chi}^{2}\left(2 m_{a}^{2}+m_{\chi}^{2}\right) \ln \left(\frac{m_{a}^{2}}{m_{\chi}^{2}}\right)}{6 m_{\chi}^{2}\left(m_{a}^{2}-m_{\chi}^{2}\right)^{4}}, \\
& D_{001}\left(p_{1}, m_{\chi}, m_{a}\right) \equiv D_{001}\left[p_{1}^{2}, p_{1}^{2}, 0,0,0, p_{1}^{2} ; 0, m_{\chi}^{2}, 0, m_{a}^{2}\right]-D_{001}\left[p_{1}^{2}, p_{1}^{2}, 0,0,0, p_{1}^{2} ; 0, m_{\chi}^{2}, m_{a}^{2}, m_{a}^{2}\right] \\
&=m_{a}^{2} \frac{-2 m_{a}^{2}+2 m_{\chi}^{2}+\left(m_{a}^{2}+m_{\chi}^{2}\right) \ln \left(\frac{m_{a}^{2}}{m_{\chi}^{2}}\right)}{12\left(m_{a}^{2}-m_{\chi}^{2}\right)^{3}}
\end{aligned}
$$

For the two-loop diagrams, the $\Pi_{G}\left(\ell^{2}\right)$ and $\Pi_{\tilde{G}}\left(\ell^{2}\right)$ in eq. (3.5) are

$$
\begin{aligned}
\Pi_{G}\left(\ell^{2}\right)= & \sum_{Q=c, b, t} g_{Q}^{2}\left(\frac{m_{Q}}{v}\right)^{2} \int_{0}^{1} d x\left[\frac{\frac{3}{2} x(1-x)}{m_{Q}^{2}-\ell^{2} x(1-x)}+m_{Q}^{2} \frac{3 x(1-x)+2\left(-1-x+x^{2}\right) c_{2 \theta_{q}}}{2\left(m_{Q}^{2}-\ell^{2} x(1-x)\right)^{2}}\right. \\
& \left.-m_{Q}^{4} \frac{1-3 x+3 x^{2}-(1-x) x c_{2 \theta_{q}}}{\left(m_{Q}^{2}-\ell^{2} x(1-x)\right)^{3}}\right] \\
\Pi_{\tilde{G}}\left(\ell^{2}\right)= & \sum_{Q=c, b, t} g_{Q}^{2}\left(\frac{m_{Q}}{v}\right)^{2} \int_{0}^{1} d x \frac{m_{Q}^{2} s_{2 \theta_{q}}}{\left(m_{Q}^{2}-\ell^{2} x(1-x)\right)^{2}} .
\end{aligned}
$$


The $F_{G}, F_{\tilde{G}}$ functions are

$$
\begin{aligned}
F_{G}\left(p_{1}^{2}, m_{\chi}^{2}, m_{a}^{2}, m_{Q}^{2}\right)= & \int_{0}^{1} d x\left[-\frac{3}{2} \frac{\partial}{\partial m_{a}^{2}} X_{1}\left(p_{1}^{2}, m_{\chi}^{2}, m_{a}^{2}, \frac{m_{Q}^{2}}{x(1-x)}\right)\right. \\
& +m_{Q}^{2} \frac{3 x(1-x)+2\left(-1-x+x^{2}\right) c_{2 \theta_{q}}}{2 x^{2}(1-x)^{2}} \frac{\partial}{\partial m_{a}^{2}} X_{2}\left(p_{1}^{2}, m_{\chi}^{2}, m_{a}^{2}, \frac{m_{Q}^{2}}{x(1-x)}\right) \\
& \left.+m_{Q}^{4} \frac{1-3 x+3 x^{2}-x(1-x) c_{2 \theta_{q}}}{x^{3}(1-x)^{3}} \frac{\partial}{\partial m_{a}^{2}} X_{3}\left(p_{1}^{2}, m_{\chi}^{2}, m_{a}^{2}, \frac{m_{Q}^{2}}{x(1-x)}\right)\right] \\
F_{\tilde{G}}\left(p_{1}^{2}, m_{\chi}^{2}, m_{a}^{2}, m_{Q}^{2}\right)= & \int_{0}^{1} d x\left[m_{Q}^{2} \frac{s_{2 \theta_{q}}}{x^{2}(1-x)^{2}} \frac{\partial}{\partial m_{a}^{2}} X_{2}\left(p_{1}^{2}, m_{\chi}^{2}, m_{a}^{2}, \frac{m_{Q}^{2}}{x(1-x)}\right)\right]
\end{aligned}
$$

where

$$
\begin{aligned}
X_{1}\left(p_{1}^{2}, m_{\chi}^{2}, m_{a}^{2}, \frac{m_{Q}^{2}}{x(1-x)}\right)= & \frac{1}{m_{a}^{2}-\frac{m_{Q}^{2}}{x(1-x)}}\left[B_{0}\left(p_{1}^{2}, m_{a}^{2}, m_{\chi}^{2}\right)-B_{0}\left(p_{1}^{2}, \frac{m_{Q}^{2}}{x(1-x)}, m_{\chi}^{2}\right)\right] \\
X_{2}\left(p_{1}^{2}, m_{\chi}^{2}, m_{a}^{2}, \frac{m_{Q}^{2}}{x(1-x)}\right)= & \frac{1}{m_{a}^{2}-\frac{m_{Q}^{2}}{x(1-x)}} \\
& \times\left[X_{1}\left(p_{1}^{2}, m_{\chi}^{2}, m_{a}^{2}, \frac{m_{Q}^{2}}{x(1-x)}\right)-C_{0}\left(p_{1}^{2}, \frac{m_{Q}^{2}}{x(1-x)}, m_{\chi}^{2}\right)\right] \\
X_{3}\left(p_{1}^{2}, m_{\chi}^{2}, m_{a}^{2}, \frac{m_{Q}^{2}}{x(1-x)}\right)= & \frac{1}{m_{a}^{2}-\frac{m_{Q}^{2}}{x(1-x)}} \\
& \times\left[X_{2}\left(p_{1}^{2}, m_{\chi}^{2}, m_{a}^{2}, \frac{m_{Q}^{2}}{x(1-x)}\right)-D_{0}\left(p_{1}^{2}, \frac{m_{Q}^{2}}{x(1-x)}, m_{\chi}^{2}\right)\right]
\end{aligned}
$$

and

$$
\begin{aligned}
& \int \frac{d^{4} \ell}{(2 \pi)^{4}} \frac{1}{\left[(\ell+p)^{2}-M^{2}\right]\left(\ell^{2}-m^{2}\right)}=\frac{i}{(4 \pi)^{2}} B_{0}\left(p^{2}, m^{2}, M^{2}\right), \\
& \int \frac{d^{4} \ell}{(2 \pi)^{4}} \frac{1}{\left[(\ell+p)^{2}-M^{2}\right]\left(\ell^{2}-m^{2}\right)^{2}}=\frac{i}{(4 \pi)^{2}} C_{0}\left(p^{2}, m^{2}, M^{2}\right), \\
& \int \frac{d^{4} \ell}{(2 \pi)^{4}} \frac{1}{\left[(\ell+p)^{2}-M^{2}\right]\left(\ell^{2}-m^{2}\right)^{3}}=\frac{i}{(4 \pi)^{2}} D_{0}\left(p^{2}, m^{2}, M^{2}\right) .
\end{aligned}
$$

Open Access. This article is distributed under the terms of the Creative Commons Attribution License (CC-BY 4.0), which permits any use, distribution and reproduction in any medium, provided the original author(s) and source are credited.

\section{References}

[1] COHERENT collaboration, Observation of Coherent Elastic Neutrino-Nucleus Scattering, Science 357 (2017) 1123 [arXiv: 1708.01294] [INSPIRE].

[2] D.Z. Freedman, Coherent Neutrino Nucleus Scattering as a Probe of the Weak Neutral Current, Phys. Rev. D 9 (1974) 1389 [InSPIRE]. 
[3] V. Brdar, W. Rodejohann and X.-J. Xu, Producing a new Fermion in Coherent Elastic Neutrino-Nucleus Scattering: from Neutrino Mass to Dark Matter, JHEP 12 (2018) 024 [arXiv: 1810.03626] [INSPIRE].

[4] J.A. Dror, G. Elor and R. Mcgehee, Directly Detecting Signals from Absorption of Fermionic Dark Matter, Phys. Rev. Lett. 124 (2020) 18 [arXiv:1905.12635] [INSPIRE].

[5] J.A. Dror, G. Elor and R. Mcgehee, Absorption of Fermionic Dark Matter by Nuclear Targets, JHEP 02 (2020) 134 [arXiv: 1908.10861] [INSPIRE].

[6] N. Hurtado, H. Mir, I.M. Shoemaker, E. Welch and J. Wyenberg, Dark Matter-Neutrino Interconversion at COHERENT, Direct Detection, and the Early Universe, Phys. Rev. D 102 (2020) 015006 [arXiv:2005.13384] [INSPIRE].

[7] J.A. Dror, G. Elor, R. McGehee and T.-T. Yu, Absorption of sub-MeV fermionic dark matter by electron targets, Phys. Rev. D 103 (2021) 035001 [arXiv:2011.01940] [InSPIRE].

[8] Y. Farzan, M. Lindner, W. Rodejohann and X.-J. Xu, Probing neutrino coupling to a light scalar with coherent neutrino scattering, JHEP 05 (2018) 066 [arXiv:1802.05171] [INSPIRE].

[9] W.-F. Chang and J. Liao, Constraints on light singlet fermion interactions from coherent elastic neutrino-nucleus scattering, Phys. Rev. D 102 (2020) 075004 [arXiv:2002.10275] [INSPIRE].

[10] D.Z. Freedman, D.N. Schramm and D.L. Tubbs, The Weak Neutral Current and Its Effects in Stellar Collapse, Ann. Rev. Nucl. Part. Sci. 27 (1977) 167 [INSPIRE].

[11] M. Drees and M. Nojiri, Neutralino-nucleon scattering revisited, Phys. Rev. D 48 (1993) 3483 [hep-ph/9307208] [INSPIRE].

[12] J. Hisano, K. Ishiwata and N. Nagata, Gluon contribution to the dark matter direct detection, Phys. Rev. D 82 (2010) 115007 [arXiv:1007.2601] [inSPIRE].

[13] S. Baek, P. Ko and P. Wu, Top-philic Scalar Dark Matter with a Vector-like Fermionic Top Partner, JHEP 10 (2016) 117 [arXiv:1606.00072] [INSPIRE].

[14] S. Baek, P. Ko and P. Wu, Heavy quark-philic scalar dark matter with a vector-like fermion portal, JCAP 07 (2018) 008 [arXiv:1709.00697] [INSPIRE].

[15] G. Arcadi, M. Lindner, F.S. Queiroz, W. Rodejohann and S. Vogl, Pseudoscalar Mediators: A WIMP model at the Neutrino Floor, JCAP 03 (2018) 042 [arXiv:1711.02110] [InSPIRE].

[16] T. Li, Revisiting the direct detection of dark matter in simplified models, Phys. Lett. B $\mathbf{7 8 2}$ (2018) 497 [arXiv: 1804.02120] [INSPIRE].

[17] T. Abe, M. Fujiwara and J. Hisano, Loop corrections to dark matter direct detection in a pseudoscalar mediator dark matter model, JHEP 02 (2019) 028 [arXiv:1810.01039] [INSPIRE].

[18] LHC Dark Matter Working Group collaboration, LHC Dark Matter Working Group: Next-generation spin-0 dark matter models, Phys. Dark Univ. 27 (2020) 100351 [arXiv: 1810.09420] [INSPIRE].

[19] T. Li and P. Wu, Simplified dark matter models with loop effects in direct detection and the constraints from indirect detection and collider search, Chin. Phys. C 43 (2019) 113102 [arXiv: 1904.03407] [INSPIRE].

[20] K.A. Mohan, D. Sengupta, T.M.P. Tait, B. Yan and C.P. Yuan, Direct Detection and LHC constraints on a t-Channel Simplified Model of Majorana Dark Matter at One Loop, JHEP 05 (2019) 115 [arXiv: 1903.05650] [INSPIRE]. 
[21] F. Ertas and F. Kahlhoefer, Loop-induced direct detection signatures from CP-violating scalar mediators, JHEP 06 (2019) 052 [arXiv: 1902.11070] [INSPIRE].

[22] F. Giacchino, A. Ibarra, L. Lopez Honorez, M.H.G. Tytgat and S. Wild, Signatures from Scalar Dark Matter with a Vector-like Quark Mediator, JCAP 02 (2016) 002 [arXiv: 1511.04452] [INSPIRE].

[23] F. Giacchino, L. Lopez-Honorez and M.H.G. Tytgat, Bremsstrahlung and Gamma Ray Lines in 3 Scenarios of Dark Matter Annihilation, JCAP 08 (2014) 046 [arXiv:1405.6921] [INSPIRE].

[24] A. Ibarra, T. Toma, M. Totzauer and S. Wild, Sharp Gamma-ray Spectral Features from Scalar Dark Matter Annihilations, Phys. Rev. D 90 (2014) 043526 [arXiv:1405.6917] [INSPIRE].

[25] S. Colucci, B. Fuks, F. Giacchino, L. Lopez Honorez, M.H.G. Tytgat and J. Vandecasteele, Top-philic Vector-Like Portal to Scalar Dark Matter, Phys. Rev. D 98 (2018) 035002 [arXiv: 1804.05068] [INSPIRE].

[26] S. Colucci, F. Giacchino, M.H.G. Tytgat and J. Vandecasteele, Radiative corrections to vectorlike portal dark matter, Phys. Rev. D 98 (2018) 115029 [arXiv:1805.10173] [INSPIRE].

[27] W. Chao, Direct detections of Majorana dark matter in vector portal, JHEP 11 (2019) 013 [arXiv: 1904.09785] [INSPIRE].

[28] X.-G. He, T. Li, X.-Q. Li, J. Tandean and H.-C. Tsai, Constraints on Scalar Dark Matter from Direct Experimental Searches, Phys. Rev. D 79 (2009) 023521 [arXiv:0811.0658] [INSPIRE].

[29] V.A. Novikov, M.A. Shifman, A.I. Vainshtein and V.I. Zakharov, Calculations in External Fields in Quantum Chromodynamics. Technical Review, Fortsch. Phys. 32 (1984) 585 [INSPIRE].

[30] M. Cirelli, E. Del Nobile and P. Panci, Tools for model-independent bounds in direct dark matter searches, JCAP 10 (2013) 019 [arXiv:1307.5955] [INSPIRE].

[31] F. Bishara, J. Brod, B. Grinstein and J. Zupan, From quarks to nucleons in dark matter direct detection, JHEP 11 (2017) 059 [arXiv:1707.06998] [INSPIRE].

[32] R.H. Helm, Inelastic and Elastic Scattering of 187-Mev Electrons from Selected Even-Even Nuclei, Phys. Rev. 104 (1956) 1466 [INSPIRE].

[33] M. Cadeddu, C. Giunti, Y.F. Li and Y.Y. Zhang, Average CsI neutron density distribution from COHERENT data, Phys. Rev. Lett. 120 (2018) 072501 [arXiv:1710. 02730] [INSPIRE].

[34] D. Aristizabal Sierra, J. Liao and D. Marfatia, Impact of form factor uncertainties on interpretations of coherent elastic neutrino-nucleus scattering data, JHEP 06 (2019) 141 [arXiv: 1902.07398] [INSPIRE].

[35] ATLAS and CMS collaborations, Measurements of the Higgs boson production and decay rates and constraints on its couplings from a combined ATLAS and CMS analysis of the LHC pp collision data at $\sqrt{s}=7$ and $8 \mathrm{TeV}$, JHEP 08 (2016) 045 [arXiv: 1606.02266] [INSPIRE].

[36] G. Bélanger, F. Boudjema, A. Pukhov and A. Semenov, Dark matter direct detection rate in a generic model with MicrOMEGAs 2.2, Comput. Phys. Commun. 180 (2009) 747 [arXiv:0803.2360] [INSPIRE].

[37] G. Bélanger, F. Boudjema, A. Goudelis, A. Pukhov and B. Zaldivar, MicrOMEGAs5.0: Freeze-in, Comput. Phys. Commun. 231 (2018) 173 [arXiv:1801.03509] [INSPIRE]. 
[38] M.J. Dolan, F. Kahlhoefer, C. McCabe and K. Schmidt-Hoberg, A taste of dark matter: Flavour constraints on pseudoscalar mediators, JHEP 03 (2015) 171 [Erratum ibid. 07 (2015) 103] [arXiv: 1412.5174] [INSPIRE].

[39] NA62 collaboration, An investigation of the very rare $K^{+} \rightarrow \pi^{+} \nu \bar{\nu}$ decay, JHEP 11 (2020) 042 [arXiv: 2007.08218] [INSPIRE].

[40] M.B. Wise, Radiatively induced flavor changing neutral higgs boson couplings, Phys. Lett. B 103 (1981) 121 [INSPIRE].

[41] H. Leutwyler and M.A. Shifman, Light Higgs Particle in Decays of $K$ and $\eta$ Mesons, Nucl. Phys. B 343 (1990) 369 [inSPIRE].

[42] ATLAS collaboration, Search for dark matter and other new phenomena in events with an energetic jet and large missing transverse momentum using the ATLAS detector, JHEP 01 (2018) 126 [arXiv:1711.03301] [INSPIRE].

[43] CMS collaboration, Search for new physics in final states with an energetic jet or a hadronically decaying $W$ or $Z$ boson and transverse momentum imbalance at $\sqrt{s}=13 \mathrm{TeV}$, Phys. Rev. D 97 (2018) 092005 [arXiv:1712.02345] [INSPIRE].

[44] ATLAS collaboration, Search for dark matter produced in association with bottom or top quarks in $\sqrt{s}=13 \mathrm{TeV}$ pp collisions with the ATLAS detector, Eur. Phys. J. C 78 (2018) 18 [arXiv: 1710.11412] [INSPIRE].

[45] ATLAS collaboration, Search for top-squark pair production in final states with one lepton, jets, and missing transverse momentum using $36 \mathrm{fb}^{-1}$ of $\sqrt{s}=13 \mathrm{TeV}$ pp collision data with the ATLAS detector, JHEP 06 (2018) 108 [arXiv:1711.11520] [INSPIRE].

[46] CMS collaboration, Searches for invisible decays of the Higgs boson in pp collisions at $\sqrt{s}=7,8$, and $13 \mathrm{TeV}$, JHEP 02 (2017) 135 [arXiv:1610.09218] [INSPIRE]. 